\title{
Effects of Add-on Ramelteon on Cognitive Impaiment in Patients with Schizophrenia: An Open-label Pilot Trial
}

\author{
Yukihiko Shirayama, Michio Takahashi, Masatoshi Suzuki, Yoshiaki Tsuruoka, Koichi Sato \\ Department of Psychiatry, Teikyo University Chiba Medical Center, Ichihara, Japan
}

\begin{abstract}
Objective: This open-label study examined the effects of ramelteon on cognitive functions in 10 outpatients with schizophrenia. Methods: Ramelteon (8 mg/day) was administered to 10 patients with schizophrenia for six months. The verbal fluency test, Trail-Making Test, the Wisconsin Card Sorting Test, the Stroop Test, the Digit Span Distraction Test, lowa Gambling Task, the Rey Auditory Verbal Learning Test were evaluated at baseline and 6 months after treatment with ramelteon.

Results: Ramelteon improved significantly the scores of Rey Auditory Verbal Learning Test. Additionally, ramelteon exerted improvements in the verbal fluency and lowa Gambling Task in 4 patients.

Conclusion: Ramelteon could be a potential therapeutic drug, in adjunctive treatment of learning and memory deficits seen in patients with schizophrenia.
\end{abstract}

KEY WORDS: Ramelteon; Schizophrenia; Verbal learning.

\section{INTRODUCTION}

Patients with schizophrenia showed verbal memory deficits, ${ }^{1,2)}$ although cognitive impairment is a core feature of schizophrenia. ${ }^{3-5)}$ The effect of atypical antipsychotic drugs on cognitive function in schizophrenia is controversial. ${ }^{6)} \mathrm{A}$ previous open-label study demonstrated that aripiprazole improved more favorably deficits in verbal learning of patients with schizophrenia over olanzapine. ${ }^{7)}$

Ramelteon is a melatonin selective agonist used to treat insomnia. Recent clinical studies reported that ramelteon was effective in maintaining mood stability for patients with bipolar disorder, ${ }^{8)}$ that ramelteon was associated with an improvement in the rating of depressive symptoms, ${ }^{9}$ that ramelteon administered elderly patients admitted for acute care provided protection against delirium, ${ }^{10)}$ and that ramelteon produced a decrease in total cholesterol and the cholesterol to high-density lipoprotein ratio in patients with schizophrenia. ${ }^{11)}$ In a recent study, melatonin ameliorated cognitive impairment induced by sleep deprivation. ${ }^{12)}$ A recent study with healthy volunteers reporting a rela-

\footnotetext{
Received: June 24, 2014 / Revised: September 12, 2014

Accepted: September 15, 2014

Address for correspondence: Yukihiko Shirayama, MD, PhD Department of Psychiatry, Teikyo University Chiba Medical Center, 3426-3 Anesaki, Ichihara 299-0111, Japan

Tel: +81-436-62-1211, Fax: +81-436-62-1511

E-mail: shirayama@rapid.ocn.ne.jp
}

tionship between melatonin and prepulse inhibition of the startle reflex, which is demonstrated to be impaired in patients with schizophrenia. ${ }^{13)}$ However, there are no reports on the effects by ramelteon on cognitive impairment in schizophrenia. Here, we have conducted an open-label study of ramelteon, added to ongoing atypical antipsychotic drug therapy, in patients with schizophrenia.

\section{METHODS}

\section{Subjects}

The participants consisted of 10 schizophrenic patients (age: $42.5 \pm 7.3$ years [mean \pm standard deviation]). All patients were recruited from the outpatient clinic of Teikyo University Chiba Medical Center. All participants met the the Diagnostic and Statistical Manual of Mental Disorders 4th edition criteria for schizophrenia, residual type, and had no other psychiatric disorders. Their psychiatric symptoms were stable for at least 3 months prior to the examination. Thus, we did not see any positive symptoms in the patients in the current study. The age at onset was $34.6 \pm 7.3$ years. The duration of illness was $94.5 \pm 64.2$ months. All participants were receiving second-generation neuroleptics, namely risperidone, quetiapine, olanzapine, blonanserin and aripiprazole (chlorpromazine equivalent dose, $760 \pm 425 \mathrm{mg} /$ day). ${ }^{14)}$ Five participants were being treated with anticholinergic drug biperiden

(c) This is an Open-Access article distributed under the terms of the Creative Commons Attribution Non-Commercial License (http://creativecommons.org/licenses/by-nc/3.0) which permits unrestricted non-commercial use, distribution, and reproduction in any medium, provided the original work is properly cited. 
(mean dose, $1.8 \pm 1.0 \mathrm{mg} /$ day). Seven were being treated with benzodiazepine drugs for anxiolytic or hypnotic effects. Participants received ramelteon at $8 \mathrm{mg}$ /day for six months. Clinically, there were no changes in symptoms between baseline and the 6-month follow up. This research was approved by the ethics committee of Teikyo University Chiba Medical Center (study \#11-32). Written informed consent was obtained after the procedure had been fully explained to each participant.

\section{Clinical Measures}

Intelligence quotient scores (IQ) were estimated from the scales of information, digit span and picture completion, using the short version of the Wechsler Adult Intelligence Scale-Revised. The mean estimated IQ was 91.0 \pm 13.5 . To assess cognitive function in the prefrontal cortex, six neuropsychological tests, the Trail-Making Test (TMT; part A and part B), the Wisconsin Card Sorting Test (Keio version; 48 cards), the verbal fluency test, the Stroop Test, the Digit Span Distraction Test (DSDT), and the Iowa Gambling Task (IGT) were administered. One memory test, the Rey Auditory Verbal Learning Test (RAVLT) was administered. Cognitive tests were evaluated at baseline and six months after treatment with ramelteon.

\section{Statistical Analysis}

The data were analyzed using paired Student's $t$-test. Differences were set to be significant when $p<0.05$. GraphPad Prizm 5 for Mac OS X (GraphPad Software, Inc., LA Jolla, CA, USA) was used.

\section{RESULTS}

The summary of cognitive function tests is shown in Table 1. The RAVLT scores of both total and recognition were significantly increased after ramelteon treatment. The RAVLT score (delayed) showed a trend for improvement: the condition of six patients improved while one patient worsened. A sample size of 10 achieves $52 \%$ power to detect a difference of -6.4 between the baseline and endpoint RAVLT (total learning) with an estimated standard deviation of 9.0 and with a significance level (alpha) of 0.05 .

Other cognition tests showed no statistical change after ramelteon treatment. However, in the verbal fluency (category), four patients improved, and one patient worsened. In the IGT, for both total and improvement, four patients showed ameliorated symptoms, while one worsened.

\section{DISCUSSION}

In this open-label study with 10 outpatients with schizophrenia, we found that ramelteon improved significantly the scores of RAVLT. RAVLT examines hippocampus- related memory functions. A recent study reviewed hippocampal dysregulation as a pathophysiological basis for psychosis in schizophrenia. ${ }^{15)}$ Next, ramelteon exerted improvements in the verbal fluency test and Iowa Gambling Task in 4 patients, but without beneficial effects on other cognitive tests such as the TMT, the Wisconsin Card Sorting Test, the Stroop Test, and the DSDT. This suggests that ramelteon exerts more effects on verbal learning and memory rather than cognitive function tests of the prefrontal cortex in patients with schizophrenia. In addition, we found that these cognitive tests were not altered by two measurements in four healthy subjects (data not shown). Therefore, it is unlikely that the improvement induced by ramelteon on cognitive function in schizophrenic patients were due to practice effects.

The biological mechanisms underlying the effect of ramelteon on memory functions in schizophrenia may be partially explained by past reports, which noted that schizophrenic patients present with lower night-time melatonin levels than healthy controls. ${ }^{16,17)}$ A recent study reported that melatonin and agomelatine, a melatonergic agonist with $5-\mathrm{HT}_{2 \mathrm{C}}$ antagonist properties, induced memory enhancing effects in the novel object test. ${ }^{18)}$ This paradigm presents a potential animal model for atypical antipsy-

Table 1. Cognitive test scores at baseline and 6 months

\begin{tabular}{lccc}
\hline \multicolumn{1}{c}{ Cognitive tests } & Baseline & 6 Months & p value \\
\hline TMT part A & $34.7 \pm 13.0$ & $35.7 \pm 17.5$ & 0.699 \\
TMT part B & $95.7 \pm 40.5$ & $88.8 \pm 51.6$ & 0.603 \\
WCST (category) & $4.80 \pm 2.09$ & $4.70 \pm 1.63$ & 0.757 \\
WCST (perserverative error) & $2.10 \pm 3.57$ & $0.80 \pm 1.87$ & 0.140 \\
Verbal fluency test (letter) & $26.0 \pm 7.1$ & $25.7 \pm 7.2$ & 0.828 \\
Verbal fluency test & $35.5 \pm 7.7$ & $39.4 \pm 7.5$ & 0.094 \\
(category) & & & \\
Stroop Test (C-D) & $7.10 \pm 4.67$ & $5.87 \pm 3.22$ & 0.237 \\
DSDT (without distractor) & $76.8 \pm 18.7$ & $80.3 \pm 15.9$ & 0.513 \\
DSDT (with distractor) & $79.7 \pm 18.1$ & $79.3 \pm 21.9$ & 0.937 \\
IGT (1-100) (total) & $-6.5 \pm 15.0$ & $3.0 \pm 22.4$ & 0.282 \\
IGT ((41-100)-(1-20)) & $-4.6 \pm 12.3$ & $5.4 \pm 20.3$ & 0.170 \\
(improvement) & & & \\
RAVLT (total learning) & $37.2 \pm 8.8$ & $43.6 \pm 9.2$ & $0.046^{*}$ \\
RAVLT (delayed recall) & $7.4 \pm 2.6$ & $9.7 \pm 2.9$ & $0.055^{\dagger}$ \\
RAVLT (recognition) & $14.3 \pm 0.9$ & $15.0 \pm 0.0$ & $0.044^{*}$ \\
\hline
\end{tabular}

Values are presented as mean \pm standard deviation.

${ }^{*} p<0.05$ (paired Student's $t$-test).

A trend for significance.

TMT, Trail-Making Test; WCST, Wisconsin Card Sorting Test; DSDT, Digit Span Distraction Test; IGT, lowa Gambling Task; RAVLT, Rey Auditory Verbal Learning Test. 
chotic activity, in the amelioration of cognitive deficits in schizophrenia. ${ }^{19)}$ Furthermore, melatonin $\mathrm{MT}_{2}$ receptordeficient mice showed learning and memory deficits in the elevated plus-maze. ${ }^{20)}$ Therefore, it is likely that ramelteon, a melatonergic agonist, works at melatonin receptor to recover impaired learning and memory on the RAVLT.

A recent study reported that aripiprazole improved verbal learning from baseline to the 8th and 26th week of assessment when compared with olanzapine. ${ }^{7)}$ Therefore, we have not ruled out the possibility that second generation antipsychotic drugs could show delayed effectiveness, although we found no difference in efficacy between the aripiprazole group, olanzapine group and other groups of antipsychotics in this study (data not shown). Future studies using larger sample sizes will be needed to address this issue.

This study has a number of limitations. First, the statistical power of is relatively small and the study lacks a control group. Additionally, practice effects can not be ruled out due to limited sample size and therefore limited power.

In conclusion, this open-label study suggests that ramelteon could be a potential therapeutic drug, in adjunctive treatment of learning and memory deficits seen in patients with schizophrenia.

\section{REFERENCES}

1. Sponheim SR, Jung RE, Seidman LJ, Mesholam-Gately RI, Manoach DS, O'Leary DS, et al. Cognitive deficits in recent-onset and chronic schizophrenia. J Psychiatr Res 2010;44:421-428.

2. Ammari N, Heinrichs RW, Miles AA. An investigation of 3 neurocognitive subtypes in schizophrenia. Schizophr Res 2010;121:32-38.

3. Elvevåg B, Goldberg TE. Cognitive impairment in schizophrenia is the core of the disorder. Crit Rev Neurobiol 2000;14:1-21.

4. Green MF. Stimulating the development of drug treatments to improve cognition in schizophrenia. Annu Rev Clin Psychol 2007;3:159-180.

5. Shirayama Y, Obata T, Matsuzawa D, Nonaka H, Kanazawa $\mathrm{Y}$, Yoshitome E, et al. Specific metabolites in the medial prefrontal cortex are associated with the neurocognitive deficits in schizophrenia: a preliminary study. Neuroimage 2010;49:2783-2790.

6. Harvey PD, McClure MM. Pharmacological approaches to the management of cognitive dysfunction in schizophrenia. Drugs 2006;66:1465-1473.
7. Kern RS, Green MF, Cornblatt BA, Owen JR, McQuade $\mathrm{RD}$, Carson $\mathrm{WH}$, et al. The neurocognitive effects of aripiprazole: an open-label comparison with olanzapine. Psychopharmacology (Berl) 2006;187:312-320.

8. Norris ER, Burke K, Correll JR, Zemanek KJ, Lerman J, Primelo RA, et al. A double-blind, randomized, placebocontrolled trial of adjunctive ramelteon for the treatment of insomnia and mood stability in patients with euthymic bipolar disorder. J Affect Disord 2013;144:141-147.

9. McElroy SL, Winstanley EL, Martens B, Patel NC, Mori N, Moeller D, et al. A randomized, placebo-controlled study of adjunctive ramelteon in ambulatory bipolar I disorder with manic symptoms and sleep disturbance. Int Clin Psychopharmacol 2011;26:48-53.

10. Hatta K, Kishi Y, Wada K, Takeuchi T, Odawara T, Usui $\mathrm{C}$, et al; DELIRIA-J Group. Preventive effects of ramelteon on delirium: a randomized placebo-controlled trial. JAMA Psychiatry 2014;71:397-403.

11. Borba CP, Fan X, Copeland PM, Paiva A, Freudenreich O, Henderson DC. Placebo-controlled pilot study of ramelteon for adiposity and lipids in patients with schizophrenia. $J$ Clin Psychopharmacol 2011;31:653-658.

12. Zhang L, Zhang HQ, Liang XY, Zhang HF, Zhang T, Liu FE. Melatonin ameliorates cognitive impairment induced by sleep deprivation in rats: role of oxidative stress, BDNF and CaMKII. Behav Brain Res 2013;256:72-81.

13. Lehtinen EK, Ucar E, Glenthøj BY, Oranje B. Effects of melatonin on prepulse inhibition, habituation and sensitization of the human startle reflex in healthy volunteers. Psychiatry Res 2014;216:418-423.

14. Woods SW. Chlorpromazine equivalent doses for the newer atypical antipsychotics. J Clin Psychiatry 2003;64:663-667.

15. Grace AA. Hippocampal dysregulation of dopamine system activity as a pathophysiological basis for psychosis in schizophrenia. Clin Psychopharmacol Neurosci 2010;8:1-9.

16. Fanget F, Claustrat B, Dalery J, Brun J, Terra JL, Marie-Cardine $\mathrm{M}$, et al. Nocturnal plasma melatonin levels in schizophrenic patients. Biol Psychiatry 1989;25:499-501.

17. Monteleone P, Natale M, La Rocca A, Maj M. Decreased nocturnal secretion of melatonin in drug-free schizophrenics: no change after subchronic treatment with antipsychotics. Neuropsychobiology 1997;36:159-163.

18. Hagiwara H, Fujita Y, Ishima T, Kunitachi S, Shirayama Y, Iyo $\mathrm{M}$, et al. Phencyclidine-induced cognitive deficits in mice are improved by subsequent subchronic administration of the antipsychotic drug perospirone: role of serotonin 5-HTlA receptors. Eur Neuropsychopharmacol 2008;18:448-454.

19. Bertaina-Anglade V, Drieu-La-Rochelle C, Mocaër E, Seguin L. Memory facilitating effects of agomelatine in the novel object recognition memory paradigm in the rat. Pharmacol Biochem Behav 2011;98:511-517.

20. Larson J, Jessen RE, Uz T, Arslan AD, Kurtuncu M, Imbesi $\mathrm{M}$, et al. Impaired hippocampal long-term potentiation in melatonin MT2 receptor-deficient mice. Neurosci Lett 2006;393:23-26. 